für, Alltagsrassismus in der praktischen Museumsarbeit sichtbar zu machen und die weiße Mehrheitsgesellschaft für Schwarze Perspektiven zu sensibilisieren.

Funding Open Access funding provided by Projekt DEAL.

Open Access Dieser Artikel wird unter der Creative Commons Namensnennung 4.0 International Lizenz veröffentlicht, welche die Nutzung, Vervielfältigung, Bearbeitung, Verbreitung und Wiedergabe in jeglichem Medium und Format erlaubt, sofern Sie den/die ursprünglichen Autor(en) und die Quelle ordnungsgemäß nennen, einen Link zur Creative Commons Lizenz beifügen und angeben, ob Änderungen vorgenommen wurden.

Die in diesem Artikel enthaltenen Bilder und sonstiges Drittmaterial unterliegen ebenfalls der genannten Creative Commons Lizenz, sofern sich aus der Abbildungslegende nichts anderes ergibt. Sofern das betreffende Material nicht unter der genannten Creative Commons Lizenz steht und die betreffende Handlung nicht nach gesetzlichen Vorschriften erlaubt ist, ist für die oben aufgeführten Weiterverwendungen des Materials die Einwilligung des jeweiligen Rechteinhabers einzuholen.

Weitere Details zur Lizenz entnehmen Sie bitte der Lizenzinformation auf http://creativecommons.org/ licenses/by/4.0/deed.de.

\title{
Bajohr, Hannes (Hrsg.): Der Anthropos im Anthropozän. Die Wiederkehr des Menschen im Moment seiner vermeintlich endgültigen Verabschiedung, 244 S., De Gruyter, Berlin u. a. 2020.
}

\section{Sina Steglich}

Online publiziert: 23. September 2020

(C) Der/die Autor(en) 2020

Das Konzept des Anthropozän wartet mit einem Paradox auf: Einerseits inthronisiert es ,den Menschen' als Namensgeber einer erdgeschichtlichen Epoche, andererseits weist es diesen selbst nicht nur als Verursacher irreversibler Veränderungen seiner Umwelt, sondern zugleich als deren prospektives Opfer aus. Nach dem Anthropos im Anthropozän zu fragen, wie es der von Hannes Bajohr zusammengestellte Band unternimmt, könnte entsprechend als Bescheidenheitsgeste aufgefasst werden, da es selbstredend im Kern des so benannten Topos liegt, sich zum Menschen zu posi-

S. Steglich $(\bowtie)$

Deutsches Historisches Institut London, London, Großbritannien

E-Mail: s.steglich@ghil.ac.uk 
tionieren. Etwas weniger bescheiden und aussichtsreicher stellt sich das Anliegen jedoch dar, wenn das Anthropozän nicht lediglich als irgendwie menschenbezogen anerkannt, vielmehr konkret danach gefragt werden soll, wie genau ,der Mensch“ wahlweise als zu dezentrierende Spezies, als existenzielle Bedrohung seiner selbst wie des Planeten oder auch als Randfigur in einem komplexen Gefüge an Organismen imaginiert und argumentativ eingesetzt wird. Entsprechend formuliert der Herausgeber eingangs die Hypothese, dass der Mensch ,,als Adressat ethischer Forderungen, als politisch Handelnder oder als Verursacher und Verantwortlicher des Klimawandels [...] weiterhin ein operativer, aber eben prekärer Begriff“" (S. 10) bleibe, den es gerade deshalb konkreter zu bestimmen gelte. Der Fokus liegt also nicht darauf, zu klären, ob und - wenn ja - inwiefern das Anthropozän adäquates Epochenkonzept, Analyseinstrument oder nützlicher Reflexionsimpuls gegenwärtiger Krisenwahrnehmungen ist, sondern es geht um das Verständnis ,des Menschen“ in seinem diskursiven Kontext. Vor diesem Hintergrund unternehmen es Vertreter_innen aus der Philosophie, den Literatur-, Kultur- und Medienwissenschaften, der Soziologie und Geschichtswissenschaft aus ihrer je spezifischen Perspektive, genau das näher zu bestimmen, worauf der Signifikant ,Mensch“ in den gegenwärtigen Diskussionen um das Anthropozän - und ihren älteren ideellen Verwandten verweist.

Dazu wird die Rede vom Anthropozän zunächst eingebettet in grundsätzliche Reflexionen über den Kern des Menschseins oder genauer: des ,Mensch-in-der-WeltSeins‘. Diesem Urgrund widmen sich die Beiträge von Joachim Fischer, Marc Rölli, Daniel Chernilo und Katharina Block, die sich explizit Deutungsangeboten der philosophischen Anthropologie zuwenden - und damit einer Disziplin, die der aktuellen Anthropozändebatte eine orientierungsstiftende historische Tiefe zu geben vermag. Zentrale Protagonisten wie Günther Anders, Arnold Gehlen, Helmuth Plessner und Max Scheler werden so nicht schlicht als Kronzeugen gegenwärtiger post- oder neohumanistischer Ansätze aufgerufen, sondern als Gesprächspartner präsentiert, denen Gehör zu schenken ist, wenn das Sprechen über das Anthropozän nicht auf der Ebene eines kernlosen Ideologems verharren oder als politische Parole primär appellativen Charakter für sich beanspruchen soll.

Ein zweiter Teil widmet sich konkreter unterschiedlichen Figuren ,des Menschen * und der damit einhergehenden Spannung zwischen individualisierenden und gattungsspezifischen Verständnissen. Menschsein im Kontext von Reproduktionsfähigkeit und Bevölkerungsdiskursen erörtert Frederike Felcht. Philip Hüpkes fragt, wie wir den Menschen denken können angesichts einer tiefenzeitlichen Skala, die sich individueller Erfahrung und Vorstellungskraft entzieht. Durchaus kontrovers erachtet Sebastian Edinger als kennzeichnend für das Anthropozän nicht etwa den Menschen, sondern Nationalstaaten und ihre geopolitischen, auf Ressourcensicherung gerichteten Interessen. Mariaenrica Giannuzzi diskutiert philosophische und ethnologische Ansätze der „Pluralisierung des Menschlichen“, indem sie Catherine Malabou und Anna Lowenhaupt Tsing vergleicht (S. 168). Schließlich stellen Christian Dries und Marie-Helen Hägele, Arantzazu Saratxaga Arregi und Stefan Färber die Perspektive der negativen Anthropologie auf das Anthropozän vor. Auch hier wird deutlich, wie stark die Debatte durch ideelle Rückbezüge geerdet werden kann. Menschsein ist demnach zu definieren über ein ,Außen“, über sein konstitutives Oszillieren zwi- 
schen Weltverbundenheit und -fremdheit sowie durch die Stellung zu einer Umwelt angesichts planetarer Endlichkeit.

Gerade im Anschluss an diese Ansätze der negativen Anthropologie will der Band mitunter bewusst erproben, den Anthropos von seinen Rändern her zu erschließen. Doch hätte die Kartierung des Themenfeldes insgesamt gewonnen, wenn die Zusammenstellung der Beiträge präziser erläutert und deren Bindekraft herausgearbeitet worden wären. Diese Chance wird auch dadurch vertan, dass es sich bei dem Beitrag des Herausgebers und dem thesenhaften Debattenausblick Dipesh Chakrabartys, die den Band rahmen, um Reprints handelt. So verharrt der Leseeindruck zuweilen auf der Ebene arbiträrer Einblicke, die stärker aufeinander bezogen und miteinander ins Gespräch hätten gebracht werden können. Ungeachtet dessen ist der vorgestellte Fragehorizont jedoch insbesondere aus Sicht der historischen Kulturwissenschaften zu begrüßen. Denn der Rekurs auf das Anthropozän macht eben nicht nur darauf aufmerksam, dass die binäre Spaltung der Welt in Natur und Kultur historisch kontingent und vor allem sehr jung ist. Vielmehr fordert er dazu auf, sich mit dem Kern des Menschseins und seinen Grenzen auseinanderzusetzen. Somit kann er zur disziplinären Selbstverständigung und -vergewisserung der Geistes- und Sozialwissenschaften beitragen, da nicht weniger als ihre anthropozentrische Begründung zur Debatte steht. Und diese, neue' Unsicherheit auch als Resultat einer nicht gar so neuen Unsicherheit des Verstehens ,des Menschen“ vorzustellen, den es gerade deshalb nicht vorschnell zu verabschieden gilt, ist der eigentliche Debattenbeitrag des Bandes.

Funding Open Access funding enabled and organized by Projekt DEAL.

Open Access Dieser Artikel wird unter der Creative Commons Namensnennung 4.0 International Lizenz veröffentlicht, welche die Nutzung, Vervielfältigung, Bearbeitung, Verbreitung und Wiedergabe in jeglichem Medium und Format erlaubt, sofern Sie den/die ursprünglichen Autor(en) und die Quelle ordnungsgemäß nennen, einen Link zur Creative Commons Lizenz beifügen und angeben, ob Änderungen vorgenommen wurden.

Die in diesem Artikel enthaltenen Bilder und sonstiges Drittmaterial unterliegen ebenfalls der genannten Creative Commons Lizenz, sofern sich aus der Abbildungslegende nichts anderes ergibt. Sofern das betreffende Material nicht unter der genannten Creative Commons Lizenz steht und die betreffende Handlung nicht nach gesetzlichen Vorschriften erlaubt ist, ist für die oben aufgeführten Weiterverwendungen des Materials die Einwilligung des jeweiligen Rechteinhabers einzuholen.

Weitere Details zur Lizenz entnehmen Sie bitte der Lizenzinformation auf http://creativecommons.org/ licenses/by/4.0/deed.de. 